\title{
Phénologie de Cola millenii K.Schum. au Bénin
}

\author{
Iboukoun Fidèle Lawin ${ }^{(1,2)}$, Kolawolé Valère Salako ${ }^{(3,4)}$, Adandé Belarmain Fandohan ${ }^{(5)}$, \\ Achille Ephrem Assogbadjo ${ }^{(2)}$, Christine Adjokè Ifètayo Nougbodé Ouinsavi ${ }^{(1)}$
}

\author{
(1) Université de Parakou. Faculté d'Agronomie. Laboratoire d'Études et de Recherches Forestières. BP 123. Parakou \\ (Bénin).E-mail : ifilawin@yahoo.fr \\ (2) Université d'Abomey-Calavi. Faculté des Sciences Agronomiques. Laboratoire d'Écologie Appliquée. 01 BP 526. \\ Cotonou (Bénin). \\ (3) Université d'Abomey-Calavi. Faculté des Sciences Agronomiques. Laboratoire de Biomathématiques et d'Estimations \\ Forestières. 04 BP 1525. Cotonou (Bénin). \\ (4) Université libre de Bruxelles. Département d'Évolution Biologique et Écologie. Av. F.D. Roosevelt, 50. CP 160/12. BE- \\ 1050 Bruxelles (Belgique). \\ (5) Université Nationale d'Agriculture. École de Foresterie Tropicale. Unité de Recherche en Foresterie et Conservation des \\ Bioressources. BP 43. Kétou (Bénin).
}

Reçu le 5 juillet 2020, accepté le 3 juin 2021, mis en ligne le 14 juin 2021.

Cet article est distribué suivant les termes et les conditions de la licence CC-BY (http://creativecommons.org/licenses/by/4.0/ deed.fr)

Description du sujet. La compréhension des facteurs influençant les phénophases des plantes d'importance socio-économique est essentielle pour leur gestion durable. Cola millenii est un arbre dont les fruits sont comestibles.

Objectifs. La présente étude a analysé les différentes phénophases de l'espèce le long d'un gradient climatique au Bénin.

Méthode. Les évènements de feuillaison, floraison et fructification ont été observés au milieu et à la fin de chaque mois (environ chaque 15 jours) durant deux ans dans huit phytodistricts distribués entre la zone guinéenne et la zone soudanoguinéenne du Bénin.

Résultats. Dans les deux zones climatiques, $C$. millenii porte des feuilles toute l'année, mais l'intensité de la feuillaison varie au cours de l'année. On observe une forte chute de feuilles de janvier à mars. Cola millenii fleurit d'octobre à avril (saison sèche). Un décalage d'un mois a été observé dans le phytodistrict du Borgou-Sud où la floraison a démarré en novembre. Le pic de la floraison a été constaté en décembre. La fructification s'étend de novembre (nouaison) à juillet (saison sèche à mi-saison pluvieuse). Un décalage d'un mois a été également observé dans le phytodistrict du Borgou-Sud où la fructification a démarré en décembre. Le pic de fructification a été observé dans la période de février à juillet. Les fruits murissent de façon progressive dans cette période. La floraison et la fructification sont positivement corrélées avec la température maximale et négativement avec l'humidité relative de l'air. De même, la floraison est négativement corrélée avec la pluviométrie.

Conclusions. Cette étude fournit des informations importantes pour une gestion durable de l'espèce dans les régions climatiques où elle est rencontrée.

Mots-clés. Floraison, fructification, gradient, pluviométrie, température, humidité relative.

\section{Phenology of Cola millenii K.Schum. in Benin}

Description of the subject. Understanding the factors influencing the phenophases of plants with socio-economic importance is essential for their sustainable management. The plant under study here, from this perspective, is Cola millenii, an edible wild fruit tree.

Objectives. This study analyzes the different phenophases of the species along a climatic gradient in Benin.

Method. Leafing, flowering, and fruiting events were observed in the middle and at the end of each month (around every 15 days) for two years in eight phytodistricts distributed across the Guinean zone and Sudano-Guinean zone of Benin.

Results. In both climatic zones, $C$. millenii bears leaves throughout the year, but the intensity of leafing varies over the year. We observed a strong fall of leaves from January to March. Cola millenii flowers from October to April (dry season). A onemonth lag was observed in the South Borgou phytodistrict, where flowering started in November. The peak of flowering was in December. Fruiting extends from November (fruit set) to July (dry season to mid-rainy season). A one-month lag was also observed in the South Borgou phytodistrict, where fruiting started in December. The peak of fruiting was 
observed from February to July. Fruits gradually ripened during this period. Flowering and fruiting were positively correlated with maximum temperature and negatively with relative humidity. Likewise, flowering was negatively correlated with rainfall. Conclusions. This study provides important information for the sustainable management of the C.millenii species in the climatic regions where it is encountered.

Keywords. Flowering, fruiting, gradient, pluviometry, temperature, relative humidity.

\section{INTRODUCTION}

La phénologie des plantes étudie les variations des phénomènes périodiques du développement végétal (Lebourgeois et al., 2006). Elle traduit les conditions écologiques récentes et permet aussi de faire un monitoring de la situation environnementale (Kouyaté, 2005). Le champ d'étude de la phénologie des plantes consiste à faire un suivi régulier des différentes étapes de leur développement et à étudier les facteurs qui l'influencent (Lebourgeois et al., 2006). En effet, plusieurs facteurs biotiques et abiotiques affectent les phénophases des plantes (Wolkovich et al., 2014). Cependant, la plupart des études indiquent que le climat (pluviométrie, température et photopériode) est un facteur essentiel affectant la phénologie des plantes en milieu tropical (Morellato et al., 2013 ; Wolkovich et al.,2014). L'étude des phénophases et des facteurs qui les influencent est donc importante pour comprendre la dynamique des populations dans les écosystèmes (Rosemartin et al., 2014). Par exemple, la période de floraison est très variable pour une même espèce parce qu'elle dépend de plusieurs facteurs dont la situation géographique de la plante (Arbonnier, 2002). Ainsi, les périodes de floraison sont souvent décalées dans le temps entre l'ouest et l'est, quand bien même les sites se trouvent sur une même isohyète (Arbonnier, 2002).

La phénologie constitue un outil pour évaluer les réponses des plantes aux changements climatiques (Rosemartin et al., 2014 ; Zouaoui et al., 2014). Â ce titre, elle apparait comme un élément clé de l'écologie des plantes car elle permet de mieux appréhender leur adaptation aux conditions climatiques (Diallo et al., 2016) et peut contribuer aux stratégies de gestion (Sinasson Sanni et al., 2017).

L'intégration des essences forestières locales intéressantes dans les programmes de valorisation des ressources locales et de la diversification agricole, notamment pour l'alimentation et la pharmacopée traditionnelle et le besoin de satisfaire les marchés grandissants, requière la connaissance des cycles phénologiques de ces essences en vue d'une bonne programmation des périodes de récolte des différents organes de ces espèces (Kouyaté, 2005).

Cola millenii K.Schum. est l'une des 50 espèces prioritaires fournissant des produits forestiers non ligneux identifiées au Bénin (Assogbadjo et al., 2017). Cette espèce est connue pour ses utilisations alimentaire, médicinale, magique, comme brosse végétale et emballage (Lawin et al., 2019). Elle préfère les forêts denses humides semi-décidues et les forêts denses sèches (observations de terrain). Elle est également rencontrée en formations saxicoles (Oumorou, 2003 ; Yedomonhan et al., 2008), en forêt galerie (Natta, 2003; Ganglo \& de Foucault, 2006), en forêt inondable (Adjakpa et al., 2011) et en forêt marécageuse (Dan, 2009 ; Adjakpa et al., 2011).

Elle est qualifiée de plante décidue par Burkill (2004), alors que Ademoh et al. (2017) indiquent qu'elle est sempervirente. Au Bénin, la floraison et la fructification de $C$. millenii s'observent en février, mars, août, novembre et décembre (Akoègninou et al., 2006). Les mois indiqués sont issus des exemplaires d'herbier et pourraient être incomplets (Akoègninou et al., 2006). Des données de suivis rigoureux de la phénologie sont donc nécessaires pour clarifier la phénologie de l'espèce et préciser le rôle probable des variables du climat.

L'objectif de cette étude est :

- de décrire les différentes phases phénologiques de C. millenii au Bénin ;

- d'analyser l'influence des variables climatiques (pluviométrie, température et humidité relative) sur le comportement phénologique de l'espèce.

\section{MATÉRIEL ET MÉTHODES}

\subsection{Milieu d'étude}

L'étude a été conduite dans huit districts phytogéographiques (Côtier, Pobè, Vallée de l'Ouémé, PlateauOuest, Plateau-Est, Zou, Bassila et Borgou-Sud) couvrant deux zones climatiques (zone guinéenne et zone soudano-guinéenne) du Bénin (Afrique de l'Ouest) (Figure 1). Ces districts phytogéographiques (encore appelés phytodistricts) ont des caractéristiques distinctes du point de vue du climat et du sol (Tableau 1). Tenant compte de la position géographique du phytodistrict du Plateau (les sites d'observation ouest et est étant distants d'au moins $140 \mathrm{~km}$ ), deux sous-phytodistricts ont été distingués, à savoir PlateauOuest et Plateau-Est.

\subsection{Collecte des données}

L'étude phénologique a été faite sur 175 individus choisis au hasard (c'est-à-dire sans tenir compte ni 


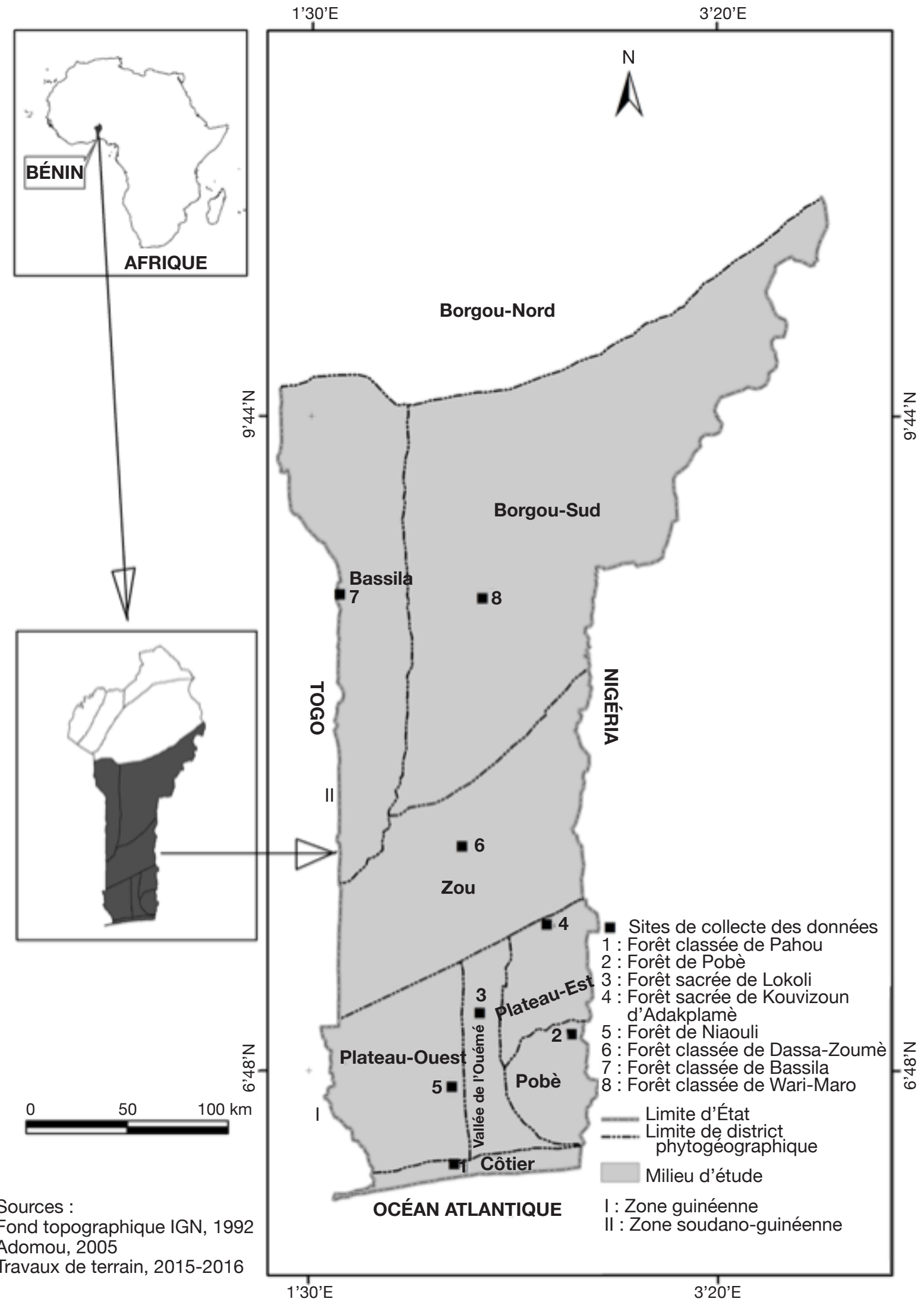

Figure 1. Milieu d'étude montrant les phytodistricts de présence de Cola millenii et les sites de collecte des données - Study area showing the phytodistricts of Cola millenii presence and the data collection sites. 
Tableau 1. Caractéristiques biophysiques des phytodistricts - Biophysical characteristics of phytodistricts (Adomou, 2011 ; Lawin et al., 2021).

\begin{tabular}{lllllll}
\hline Zone climatique & Phytodistrict & $\begin{array}{l}\text { Pluviométrie moyenne } \\
(\mathrm{mm})\end{array}$ & $\begin{array}{l}\text { Tmin } \\
\left({ }^{\circ} \mathrm{C}\right)\end{array}$ & $\begin{array}{l}\text { Tmax } \\
\left({ }^{\circ} \mathrm{C}\right)\end{array}$ & $\begin{array}{l}\text { Hmoy } \\
(\%)\end{array}$ & Type de sol \\
\hline Guinéenne & Côtier & 1351 & 24 & 30 & 79 & $\begin{array}{l}\text { Sableux } \\
\text { Pobè }\end{array}$ \\
& 1138 & 23 & 32 & 73 & $\begin{array}{l}\text { Sol ferrallitique } \\
\text { sans concrétions }\end{array}$ \\
& Vallée de l'Ouémé & 1138 & 23 & 32 & 73 & $\begin{array}{l}\text { Sol hydromorphe } \\
\end{array}$ \\
& Plateau (Est et Ouest) & $1138-1351$ & $23-24$ & $30-32$ & $73-79$ & $\begin{array}{l}\text { Sol ferrallitique } \\
\text { sans concrétions }\end{array}$ \\
\hline Soudano-guinéenne & Zou & 1081 & 22 & 33 & 68 & Sol ferrugineux \\
& Bassila & 1200 & 21 & 33 & 59 & Sol ferrugineux \\
& Borgou-Sud & 1164 & 21 & 32 & 61 & Sol ferrugineux \\
\hline
\end{tabular}

Tmin : température minimale - minimum temperature ; Tmax : température maximale - maximum temperature ; Hmoy : humidité relative moyenne - average relative humidity ; les moyennes ont été calculées à partir d'une série de données de 31 ans (1986-2016) obtenues à Météo-Bénin - averages were calculated from a 31-year data series (1986-2016) obtained from Meteo-Benin.

de leur grosseur, ni de leur hauteur), à raison de 25 par phytodistrict. Les arbres choisis dans une même forêt sont distants l'un de l'autre d'au moins $100 \mathrm{~m}$. Ces arbres sélectionnés $(10,03 \leq \mathrm{DBH} \leq 67,32 \mathrm{~cm})$ ont déjà produit au moins une fois des fruits avant le début du suivi phénologique. Frankie et al. (1974) suggèrent trois conditions idéales pour une étude phénologique :

- station relativement peu perturbée, à l'abri de pressions (coupes, feux de végétation, etc.) susceptibles d'entrainer une modification de la structure de la population ;

- effectif élevé, pouvant représenter la population considérée afin d'établir les variations au sein des populations de l'espèce ;

- suivi phénologique pendant plusieurs années afin d'établir la périodicité des différentes phases.

Les sites de collecte des données ont été choisis en tenant compte de trois critères, à savoir :

- le statut protégé de la forêt: ce choix a été fait pour limiter l'influence des pressions anthropiques sur les évènements phénologiques de l'espèce ;

- la grandeur de la forêt : ce critère a été choisi par souci de représentativité ;

- la densité élevée de l'espèce dans la forêt : ce critère a été pris en compte pour avoir la possibilité de choisir des arbres distants l'un de l'autre d'au moins $100 \mathrm{~m}$.

Ainsi, le suivi phénologique a été effectué pendant deux ans (janvier 2015 à décembre 2016) suivant le protocole de Kouyaté (2005) et en tenant compte des conditions suggérées par Frankie et al. (1974). Sur chaque arbre, les données enregistrées sont la présence ou l'absence de feuilles, d'inflorescences et de fruits (Ratovonamana et al., 2011; Alvarado et al., 2014). Les observations ont été effectuées deux fois par mois précisément au milieu et à la fin de chaque mois, soit environ chaque 15 jours. Chaque arbre a été étiqueté (Ratovonamana et al., 2011 ; Alvarado et al., 2014) à l'aide d'un tissu blanc pour faciliter son identification et éviter qu'il ne soit mutilé.

La feuillaison est la période allant de la formation des bourgeons foliaires au jaunissement des feuilles sur pied et/ou de leur chute. Elle est marquée par un stade clé telle que la pleine feuillaison (abondance de feuilles) qui correspond à la période pendant laquelle plus de $50 \%$ de feuilles vertes sont observées sur les arbres. S'étendant de la formation des bourgeons floraux jusqu'au dessèchement et/ou la chute des fleurs, la floraison est marquée par un stade clé (la pleine floraison) correspondant à une période où plus de $50 \%$ des fleurs d'un arbre s'épanouissent. La fructification s'étend de la nouaison (début de la formation des fruits) jusqu'à la maturation (moment où la couleur des fruits passe du vert au rouge, rouge-orange ou jaunâtre). Chacune des phases est caractérisée par cinq stades phénologiques (Tableau 2) comme proposés par Grouzis \& Sicot (1980). Les observations ont été effectuées à l'œil nu (observation visuelle et directe) et parfois à l'aide de jumelles pour la cime des arbres. Tous les stades de chacune des trois phénophases ont été observés. Les données climatiques mensuelles (pluviométrie, température minimale, température maximale et humidité relative de l'air) de la période d'observation ont été recueillies auprès du service météorologique du Bénin (Météo-Bénin) pour chacune des localités et ont permis d'examiner la corrélation entre le climat et les phénophases de feuillaison, de floraison et de fructification de $C$. millenii au Bénin. Ces données sont celles des stations météorologiques les plus proches des sites d'observations phénologiques. Il s'agit des stations de Cotonou, Bohicon, Savè, Natitingou et Parakou. 
Tableau 2. Phénophases, stades et caractéristiques correspondantes reconnues pour le suivi phénologique des espèces végétales (adapté de Grouzis \& Sicot, 1980) - Phenophases, stages and corresponding characteristics recognized for the phenological monitoring of plant species (adapted from Grouzis \& Sicot, 1980).

\begin{tabular}{|c|c|c|}
\hline Phénophase & Stade & Caractéristiques \\
\hline \multirow[t]{5}{*}{ Feuillaison } & Fe1 & Bourgeons foliaires et/ou anciennes feuilles \\
\hline & $\mathrm{Fe} 2$ & Bourgeons foliaires + feuilles épanouies (plus de $10 \%$ et moins de $50 \%$ des rameaux de l'individu) \\
\hline & $\mathrm{Fe} 3$ & Feuilles en majorité épanouies \\
\hline & $\mathrm{Fe} 4$ & $\begin{array}{l}\text { Feuilles vertes et feuilles sèches ou ayant changé de couleur (supérieures à } 10 \% \text { et inférieures à } \\
50 \% \text { ) }\end{array}$ \\
\hline & $\mathrm{Fe} 5$ & $\begin{array}{l}\text { Les rameaux de l'individu de l'espèce ayant des feuilles sèches ou jaunes sont au-delà de } 50 \% \\
\text { et/ou chute des feuilles }\end{array}$ \\
\hline \multirow[t]{5}{*}{ Floraison } & Fl1 & Seuls les bourgeons floraux sont présents \\
\hline & $\mathrm{Fl} 2$ & Présence de bourgeons floraux et fleurs épanouies (supérieurs à 10 \% et inférieurs à 50 \%) \\
\hline & $\mathrm{Fl3}$ & Présence de fleurs épanouies sur plus de la moitié des rameaux \\
\hline & Fl4 & Fleurs épanouies et fleurs sèches (supérieures à 10 \% et inférieures à 50 \%) \\
\hline & $\mathrm{Fl5}$ & La plupart des fleurs sont sèches ; chute des fleurs \\
\hline \multirow[t]{5}{*}{ Fructification } & Fr1 & Nouaison \\
\hline & Fr2 & Phase de développement du jeune fruit, évolution vers la taille normale \\
\hline & Fr3 & Plus de $50 \%$ des fruits ont atteint la taille normale \\
\hline & Fr4 & Fruit mûr et possibilité de récolte \\
\hline & Fr5 & Fruit entièrement mûr sur l'arbre \\
\hline
\end{tabular}

\subsection{Analyse des données}

La proportion (en \%) d'individus dans chaque phase et chaque stade de feuillaison, de floraison et de fructification a été calculée par mois. Des diagrammes phénologiques concernant les différentes phases ont été réalisés pour tout le milieu d'étude, par zone climatique et par phytodistrict pour illustrer la variation de cette proportion au cours des deux ans. Par rapport aux différents stades, un diagramme montrant la situation globale du milieu d'étude a été réalisé. Pour examiner la corrélation de chaque phénophase avec les variables climatiques, le coefficient de corrélation de Spearman a été utilisé. La significativité de la valeur du coefficient de corrélation a été testée dans le package «Hmisc» (Harrell et al., 2018) du logiciel R 3.3.2. (R Core Team, 2018).

\section{RÉSULTATS}

\subsection{Phases et stades phénologiques de $C$. millenii}

Dans le milieu d'étude, C. millenii porte des feuilles toute l'année (Figure 2). On note la présence simultanée de bourgeons foliaires et d'anciennes feuilles tout au long de l'année (Figure 3a). La pleine feuillaison se déroule d'avril à décembre (Figure 3a).

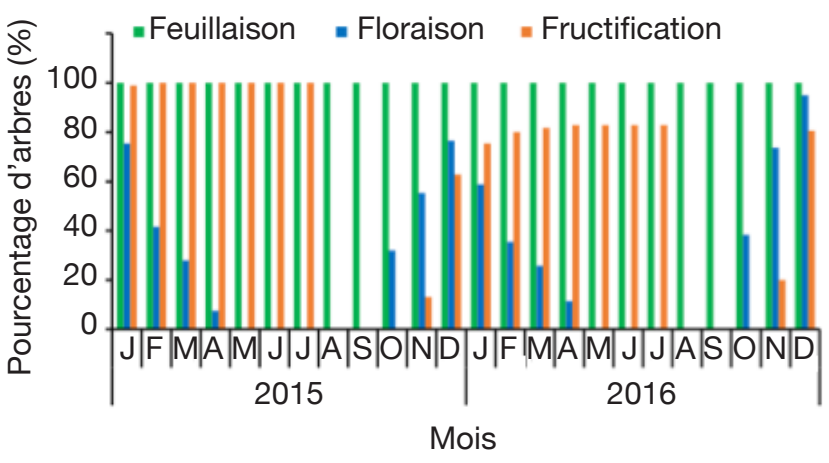

Figure 2. Diagramme phénologique de Cola millenii de 2015 à 2016 dans la zone d'étude - Phenological diagram of Cola millenii from 2015 to 2016 in the study area.

On observe une forte chute de feuilles (stade Fe 5 : les rameaux de l'individu de l'espèce ayant des feuilles sèches ou jaunes sont au-delà de $50 \%$ et/ou chute des feuilles) de janvier à mars dans tout le milieu d'étude (Figure 3a). L'arbre perd parfois plus de $80 \%$ de ses anciennes feuilles au cours de cette période.

L'entrée dans les mêmes phases phénologiques (floraison et fructification) ne se réalise pas au même moment au niveau de tous les arbres (Figure 2). Par exemple, $32 \%$ des arbres sont entrés en floraison en octobre 2016, alors que ce taux a atteint $76,57 \%$ en décembre. 

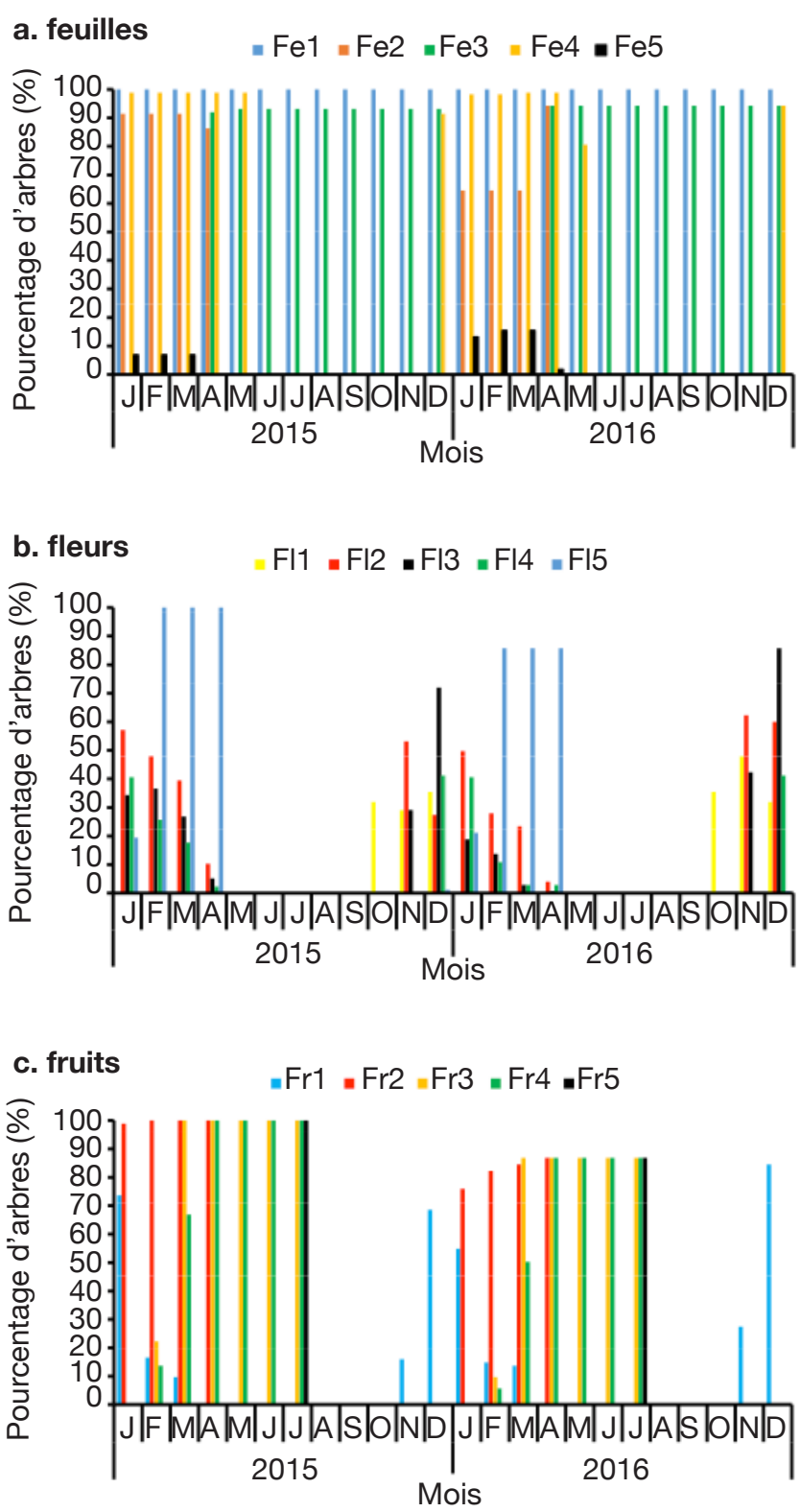

Figure 3. Stades phénologiques globaux de Cola millenii de 2015 à 2016 - Global phenological stages of Cola millenii from 2015 to 2016.

Cola millenii fleurit d'octobre à avril (Figure 2). La période d'abondance de floraison (stade $\mathrm{Fl} 3$ : présence de fleurs épanouies sur plus de la moitié des rameaux) est remarquée en décembre (Figure 3b).

La période de fructification de C. millenii s'étend de novembre (nouaison) à juillet (Figure 2). La période d'abondance de fructification (stade Fr3 : plus de $50 \%$ des fruits ont atteint la taille normale), quant à elle, commence à partir de février et s'étend jusqu'à juillet (Figure 3c), et les fruits murissent de façon progressive dans la même période (Figure 3c). Une fois mûrs, les fruits peuvent rester sur l'arbre, en bon état, jusqu'à trois mois avant de se détériorer. a. zone guinéenne

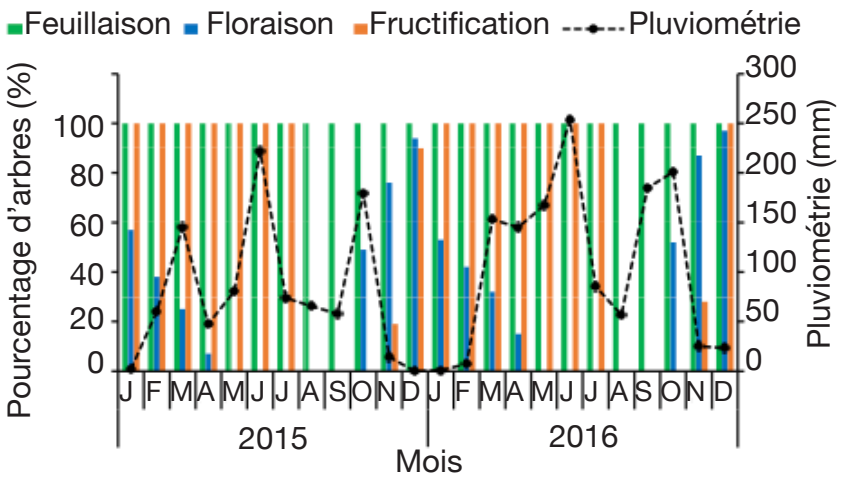

\section{b. zone soudano-guinéenne}

„ Feuillaison = Floraison „ Fructification -...-. Pluviométrie

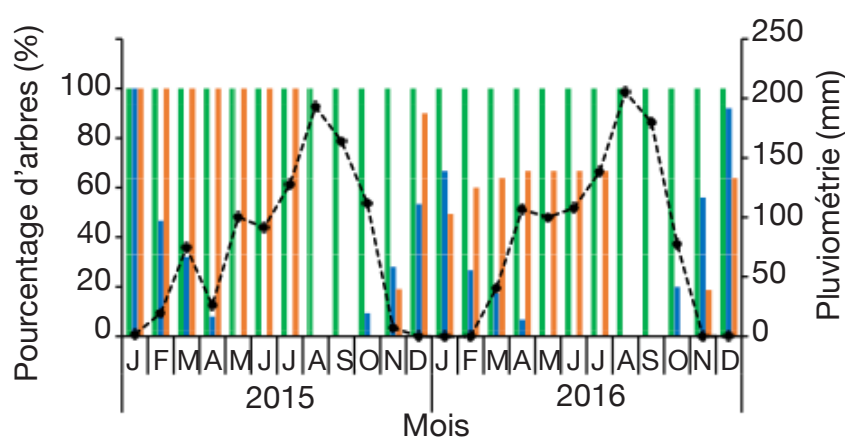

Figure 4. Diagramme phénologique de Cola millenii de 2015 à 2016 dans les zones climatiques - Phenological diagram of Cola millenii from 2015 to 2016 in climatic zones.

\subsection{Influence des conditions climatiques sur le comportement phénologique de $C$. millenii}

Les pourcentages d'arbres dans les différentes phases de floraison et fructification varient d'une zone climatique à une autre, d'un phytodistrict à un autre et d'une année à une autre (Figures 4 et 5). Dans la période d'octobre 2015 à avril 2016, il a été observé une tendance à la baisse du pourcentage d'arbres en floraison suivant le gradient sud-nord, c'est-à-dire de la zone guinéenne (Figure 4a) à la zone soudanoguinéenne (Figure 4b). La floraison démarre en octobre, sauf dans le phytodistrict du Borgou-Sud où elle démarre en novembre et prend fin en avril (Figure 5b), soit un mois de décalage. Contrairement à l'ensemble des phytodistricts où la fructification a démarré en novembre, c'est plutôt en décembre qu'elle a été constatée dans le phytodistrict du BorgouSud (Figure 5b). La floraison et la fructification chevauchent deux années. Mais c'est surtout en saison sèche (novembre à février) que l'espèce fleurit. La fructification démarre en saison sèche et se poursuit en saison des pluies. Au cours de la période d'octobre 2015 à avril 2016, la plupart des arbres n'ont pas fleuri 


\section{a. Bassila}

- Feuillaison - Floraison $=$ Fructification ...... Pluviométrie

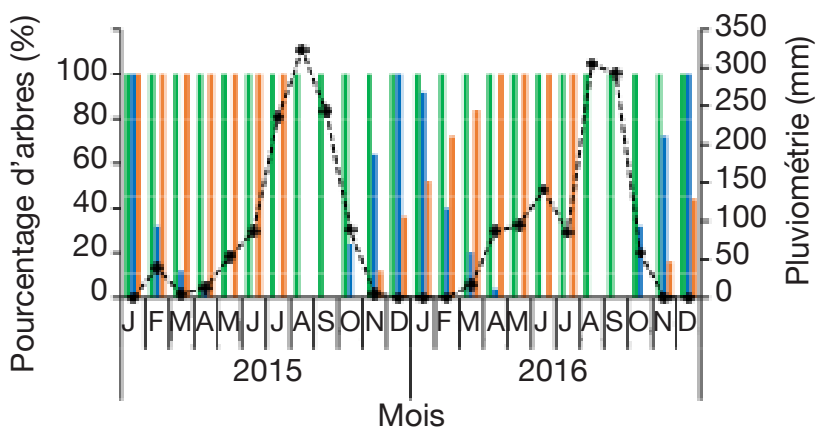

\section{c. Côtier}

=Feuillaison = Floraison $=$ Fructification--.... Pluviométrie

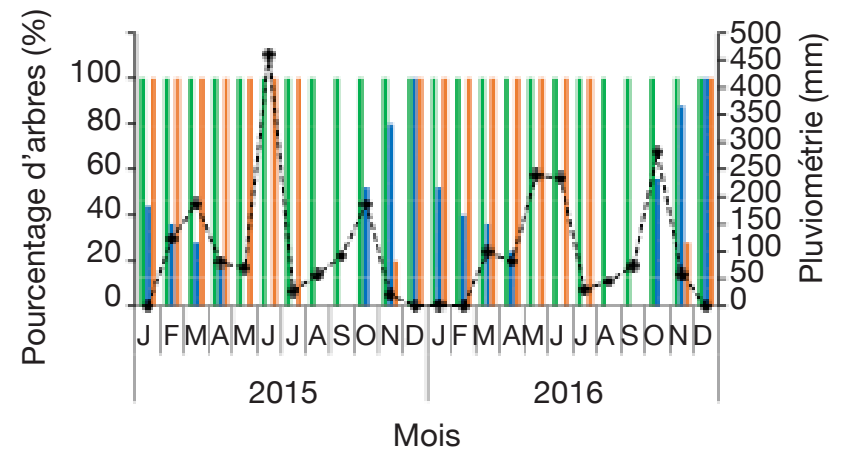

e. Plateau-Ouest

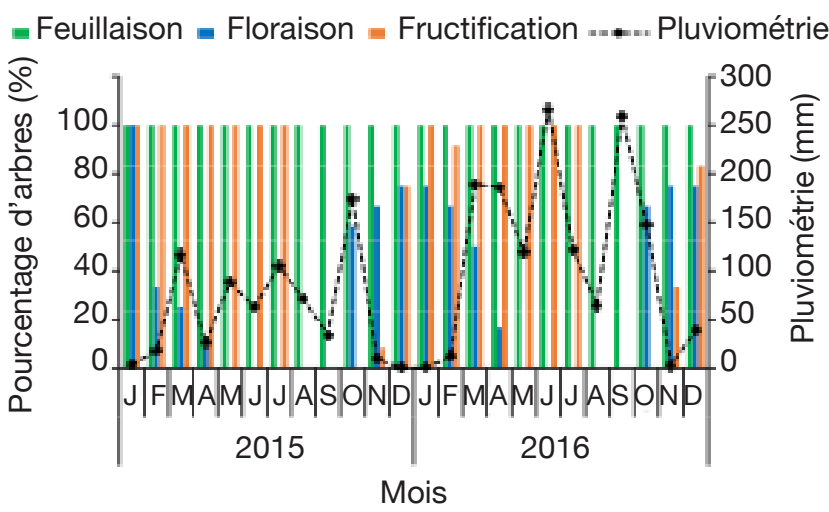

\section{g. Vallée de l'Ouémé}

- Feuillaison $=$ Floraison $=$ Fructification $\ldots$-... Pluviométrie

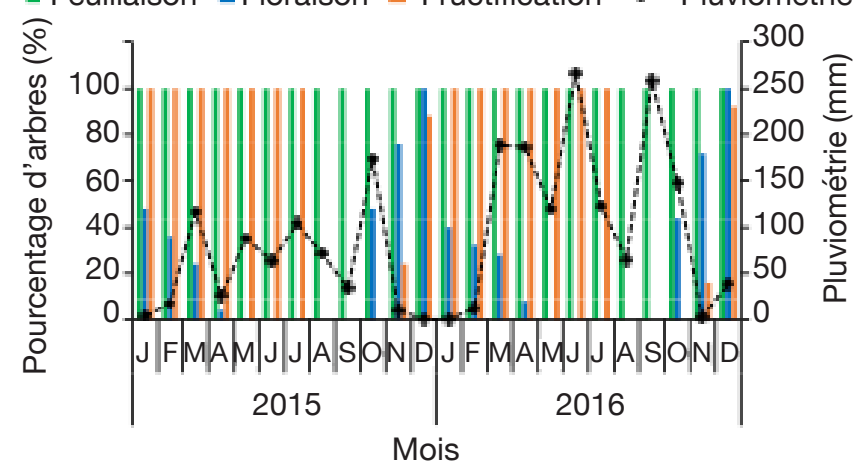

\section{b. Borgou-Sud}

- Feuillaison = Floraison = Fructification -..... Pluviométrie

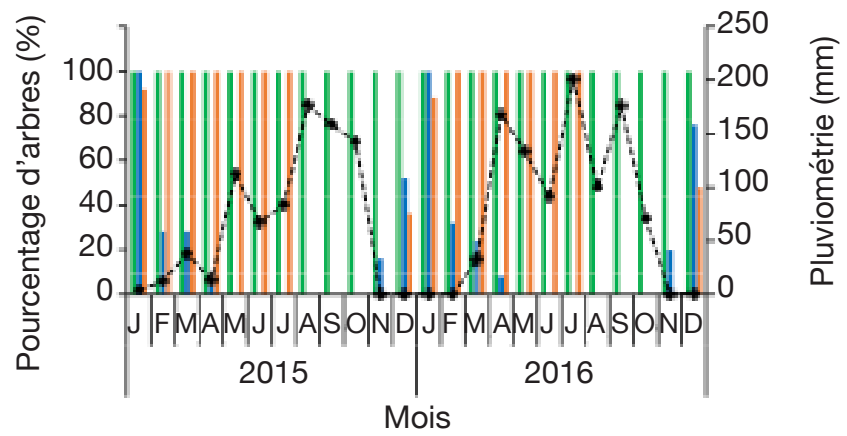

\section{d. Plateau-Est}

= Feuillaison $=$ Floraison $=$ Fructification ......Pluviométrie

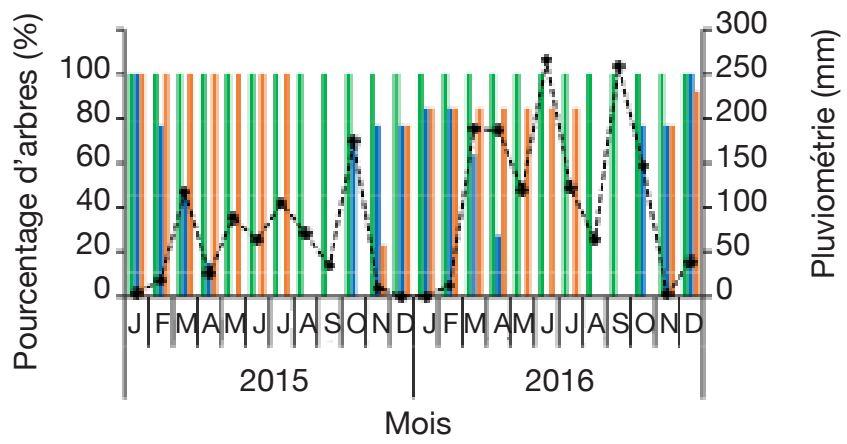

\section{f. Pobè}

= Feuillaison $=$ Floraison $=$ Fructification ......Pluviométrie
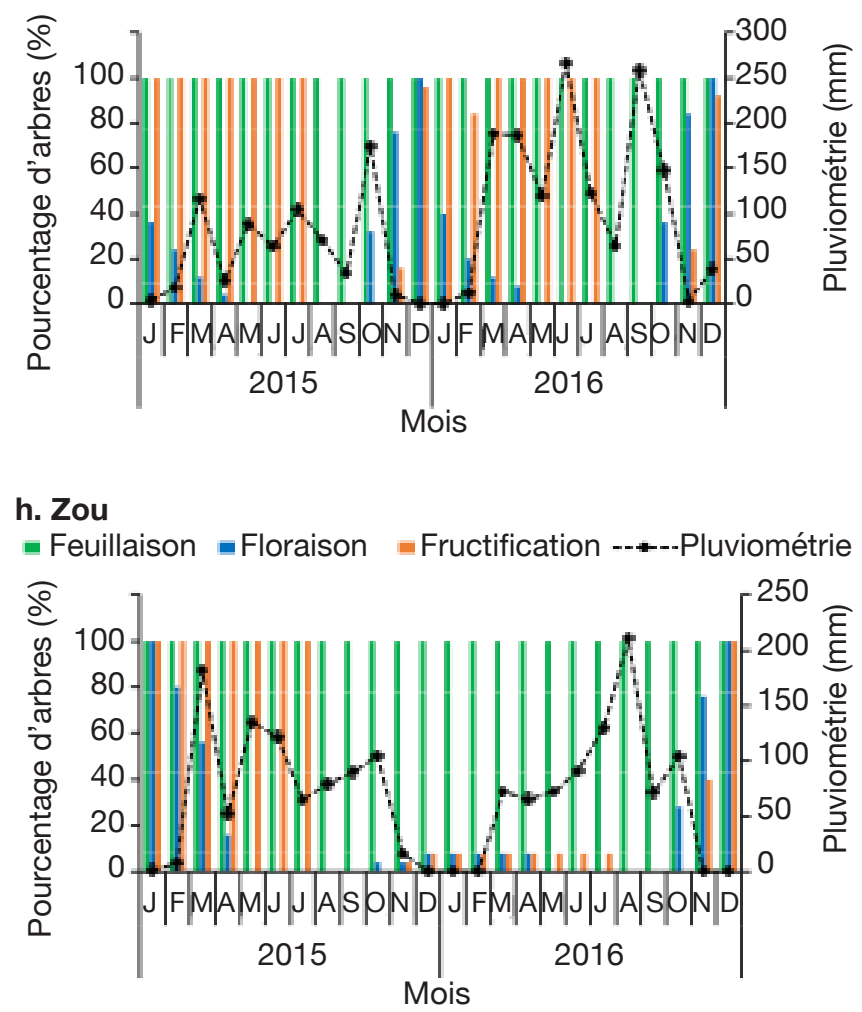

Figure 5. Diagramme phénologique de Cola millenii de 2015 à 2016 dans les phytodistricts - Phenological diagram of Cola millenii from 2015 to 2016 in the phytodistricts. 
et n'ont donc pas fructifié dans le phytodistrict du Zou (Figure 5h).

Dans les deux zones climatiques, des corrélations négatives et significatives (Tableau 3) ont été observées entre la pluviométrie moyenne mensuelle et la floraison, entre l'humidité relative moyenne mensuelle et la floraison et entre l'humidité relative moyenne mensuelle et la fructification. Par contre, des corrélations positives et significatives (Tableau 3) ont été notées d'une part entre la température maximale et la floraison et, d'autre part, entre la température maximale et la fructification.

\section{DISCUSSION}

\section{1. Évènements phénologiques de $C$. millenii}

Cola millenii porte des feuilles toute l'année. Le même résultat a été obtenu par Ademoh et al. (2017) au Nigéria sur la même espèce et d'autres espèces du même genre dont Cola nitida et Cola acuminata. La perte de plus de $80 \%$ des anciennes feuilles de l'espèce par moment suggère qu'une température très élevée dans une localité pourrait entrainer la défeuillaison totale des arbres de l'espèce dans les endroits où la réserve en eau du sol est faible.

Tous les arbres n'entrent pas au même moment dans les mêmes phases phénologiques (floraison et fructification). Des variations similaires ont été constatées sur plusieurs plantes dont Acacia tortillis subsp. raddiana (Savi) Brenan au Sénégal (Diallo et al., 2016), Ziziphus lotus (L.) Lam. en Tunisie (Zouaoui et al., 2014), Ficus mollis Vahl en Inde (Yadav \& Yadav, 2008) et Spondias mombin L. au Panama (Adler \& Kielpinski, 2000). La variabilité des phénophases entre les individus correspond à une aptitude propre à chaque espèce dans la conquête du milieu (Seghieri \& Simier, 2002). La pente, la disponibilité en eau du sol (Lobo et al., 2003 ; Mahamane et al., 2007), la nature du sol et l'altitude (Diouf \& Zaafouri, 2003 ; Jaouadi et al., 2012 ; Zouaoui et al., 2014) pourraient justifier ces constats.

Cette étude a révélé que l'espèce fleurit d'octobre à avril et que la période de fructification s'étend de novembre à juillet. Cependant, la flore analytique du Bénin indique que la floraison et la fructification de l'espèce se déroulent en février, mars, août, novembre et décembre (Akoègninou et al., 2006), mais les mois mentionnés ont été relevés à partir d'exemplaires d'herbiers et ne sont donc pas exhaustifs (Akoègninou et al., 2006). Par contre, au sud-ouest du Nigéria, la floraison de $C$. millenii est observée de janvier à mars et la fructification est enregistrée de février à mai (Adebola \& Morakinyo, 2006). Ces variations dans les phénophases de l'espèce pourraient être liées aux conditions environnementales locales (Wolkovich et al., 2014).

Tableau 3. Corrélation de Spearman entre les variables climatiques et les phénophases de Cola millenii - Spearman correlation coefficients between climatic variables and phenophases of Cola millenii.

\begin{tabular}{|c|c|c|c|c|c|c|}
\hline \multirow[t]{2}{*}{ Variables climatiques } & \multicolumn{2}{|c|}{ Feuillaison } & \multicolumn{2}{|c|}{ Floraison } & \multicolumn{2}{|c|}{ Fructification } \\
\hline & Coeff. & $p$-value & Coeff. & $p$-value & Coeff. & $p$-value \\
\hline \multicolumn{7}{|l|}{ Zone guinéenne } \\
\hline Pluviométrie moyenne mensuelle & - & - & $-0,465$ & $8,65-08$ & $-0,041$ & 0,65 \\
\hline Température maximale & - & - & 0,568 & $1,27-11$ & 0,221 & 0,01 \\
\hline Température minimale & - & - & 0,053 & 0,56 & 0,151 & 0,09 \\
\hline Humidité relative moyenne mensuelle & - & - & $-0,651$ & $7,66-16$ & $-0,326$ & 0,00 \\
\hline \multicolumn{7}{|l|}{ Zone soudano-guinéenne } \\
\hline Pluviométrie moyenne mensuelle & - & - & $-0,753$ & $2,22-14$ & $-0,171$ & 0,15 \\
\hline Température maximale & - & - & 0,608 & $1,46-08$ & $\mathbf{0 , 3 3 3}$ & 0,00 \\
\hline Température minimale & - & - & $-0,307$ & 0,08 & 0,183 & 0,12 \\
\hline Humidité relative moyenne mensuelle & - & - & $-0,771$ & $2,29-15$ & $-0,379$ & 0,00 \\
\hline
\end{tabular}

Les valeurs en gras indiquent que les coefficients de corrélation sont significatifs au seuil de $5 \%$ - values in bold indicate that the correlation coefficients are significant at the $5 \%$ level ; - : il n'a pas été possible de calculer le coefficient de corrélation entre les variables climatiques et la feuillaison parce que le pourcentage d'arbre en feuillaison ne varie pas - it was not possible to calculate the correlation coefficient between the climatic variables and the leafing because the percentage of tree in leafing does not vary; l'écarttype est nul, ne permettant pas de calculer ledit coefficient - the standard deviation is zero, which does not allow the calculation of correlation coefficient. 


\subsection{Conditions climatiques et comportement phénologique de $C$. millenii}

La proportion d'arbres dans les différentes phases de floraison et fructification varie d'une zone climatique à une autre et d'une année à une autre conformément aux résultats de plusieurs études (Adler \& Kielpinski, 2000 ; Yadav \& Yadav, 2008 ; Zouaoui et al., 2014). Ceci peut s'expliquer par la variabilité climatique inter-zone et inter-annuelle. Le pourcentage d'arbres en floraison a diminué suivant le gradient sud-nord d'octobre 2015 à avril 2016. Cela pourrait être dû au fait que la floraison est une phase très dépendante des facteurs environnementaux (Jaouadi et al., 2012). De façon spécifique, il a été observé au cours de la période d'octobre 2015 à avril 2016 (moment habituel de floraison) une faible proportion d'arbres en phase de floraison et de fructification dans le phytodistrict du Zou (en zone soudano-guinéenne), comparativement aux autres phytodistricts, traduisant une variabilité des phénophases d'une localité à une autre. Ce résultat est similaire aux observations faites sur Tamarindus indica L. dans la même zone (Fandohan et al., 2015). Ceci peut s'expliquer non seulement par l'humidité du sol et la topographie (Lobo et al., 2003), mais aussi par la variabilité des conditions climatiques. En effet, les populations de $C$. millenii rencontrées dans le phytodistrict du Zou se trouvent sur les collines où l'humidité du sol pourrait constituer un facteur limitant pour la floraison et la fructification.

Dans les deux zones climatiques, la floraison est négativement corrélée avec la pluviométrie et l'humidité relative de l'air, mais positivement avec la température maximale. Autrement dit, le pourcentage d'arbres en floraison diminue avec une augmentation de la pluviométrie et de l'humidité relative de l'air, mais augmente avec une augmentation de la température maximale. De même, la fructification est positivement corrélée avec la température maximale et négativement avec l'humidité relative de l'air, ce qui veut dire que le pourcentage d'arbres en fructification augmente avec une augmentation de la température maximale mais diminue avec une augmentation de l'humidité relative de l'air. Ceci suggère que la température, les précipitations et l'humidité relative de l'air sont des déterminants importants de la floraison et de la fructification (Crimmins et al., 2011 ; Morellato et al., 2013). Ces résultats sont conformes à ceux de Okullo et al. (2004) sur Vitellaria paradoxa subsp. nilotica (Kotschy) A.N.Henry, Chithra \& N.C.Nair en Ouganda. En effet, il faut un certain niveau de précipitation et de température pour qu'un arbre fructifie (Rasamimanana et al., 2012). La forte chute de feuilles observées de janvier à mars (saison sèche) indique qu'une diminution de la pluviométrie entraine une augmentation de la défoliation conformément aux travaux de Ratovonamana et al. (2011) sur plusieurs espèces d'arbres (Adansonia rubrostipa Jum. \& H.Perrier, Cedrelopsis gracilis J.-F.Leroy, Didierea madagascariensis Baill., Operculicarya decaryi H.Perrier, Poupartia minor (Bojer) L. Marchand, etc.) au sud-ouest de Madagascar.

\section{CONCLUSIONS}

Les travaux de cette étude ont permis de fournir des informations utiles sur les différentes phénophases de l'espèce. Les observations successives ont révélé que C. millenii porte des feuilles toute l'année. Toutefois, le taux de couverture en feuilles varie au cours de l'année. Des variations ont aussi été observées au cours des phases de floraison et de fructification entre zones climatiques, districts phytogéographiques et années, dénotant en partie l'effet des facteurs abiotiques. Les corrélations significatives entre les phénophases et les conditions climatiques confortent cette thèse. Cette étude n'a pas pris en compte l'effet potentiel du diamètre des arbres ainsi que du type de sol qui sont aussi des déterminants importants de la phénologie de l'espèce. Néanmoins, la connaissance de la période de floraison et de fructification rapportée dans cette étude pourra permettre aux décideurs et aux populations locales de prendre la décision de réaliser des pare-feux pendant ladite période ou avant celle-ci autour de l'habitat de l'espèce. Cette activité devrait permettre d'éviter la destruction des fleurs par les feux de végétation incontrôlés, contribuer à maintenir une bonne fructification de l'espèce dans son habitat au profit des populations et protéger les ressources naturelles associées.

\section{Remerciements}

Cette recherche a fait l'objet d'un soutien de la Fondation Internationale pour la Science (Suède), par l'intermédiaire d'une bourse de recherche accordée à Iboukoun Fidèle Lawin (Grant no D/5875-1). Kolawolé Valère Salako a bénéficié du soutien de Wallonie-Bruxelles International Belgique, à travers la bourse d'excellence post-doctorale (Bourse no SUB/2019/443681). Nous remercions Bienvenu Dossa pour son aide.

\section{Bibliographie}

Adebola P.O. \& Morakinyo J.A., 2006. Evaluation of morpho-agronomic variability of wild and cultivated kola (Cola species Schott et Endel.) in South Western Nigeria. Genet. Resour. Crop Evol., 53, 687-694, doi. org/10.1007/s10722-004-3558-1

Ademoh F.O., Muoghalu J.I. \& Onwumere B., 2017. Temporal pattern of tree community dynamics in a 
secondary forest in southwestern Nigeria, 29 years after a ground fire. Global Ecol. Conserv., 9, 148-170, doi. org/10.1016/j.gecco.2016.11.005

Adjakpa J.B. et al., 2011. Diversité du peuplement ligneux d'une forêt dense en zone sub-humide : cas de la forêt de Sakété dans le sud-Bénin en Afrique de l'Ouest. Int. J. Biol. Chem. Sci., 5(6), 2291-2305, doi.org/10.4314/ ijbes.v5i6.10

Adler G.H. \& Kielpinski K.A., 2000. Reproductive phenology of a tropical canopy tree, Spondias mombin. Biotropica, 32(4a), 686-692, doi.org/10.1646/00063606(2000)032[0686:rpoatc]2.0.co;2

Adomou A., 2011. Phytogéographie du Bénin/ Phytogeography of Benin. In: Neuenschwander P., Sinsin B. \& Goergen G., eds. Conservation de la nature en Afrique de l'Ouest: une liste rouge pour le Bénin/ Nature conservation in West Africa: red list for Benin. Ibadan, Nigeria: International Institute of Tropical Agriculture, 14-20.

Akoègninou A. et al., 2006. Flore analytique du Bénin. Leiden, The Netherlands: Backhuys Publishers.

Alvarado S. et al., 2014. Fire and the reproductive phenology of endangered Madagascar sclerophyllous tapia woodlands. S. Afr. J. Bot., 94, 79-87, doi.org/10.1016/j. sajb.2014.06.001

Arbonnier M., 2002. Arbres, arbustes et lianes des zones sèches d'Afrique de l'Ouest. Paris : CIRAD-MNHN.

Assogbadjo A.E. et al., 2017. Diversity and prioritization of non-timber forest products for economic valuation in Benin (West Africa). Afr. J. Rural Dev., 2(1), 105-115.

Burkill H.M., 2004. The useful plants of West Tropical Africa. Kew, UK: Royal Botanic Gardens.

Crimmins T.M., Crimmins M.A. \& Bertelsen C.D., 2011. Onset of summer flowering in a 'Sky Island' is driven by monsoon moisture. New Phytol., 191, 468-479, doi. org/10.1111/j.1469-8137.2011.03705.x

Dan C., 2009. Études écologique, floristique, phytosociologique et ethnobotanique de la forêt marécageuse de Lokoli; Zogbodomey-Bénin. Thèse de doctorat: Université libre de Bruxelles (Belgique).

Diallo M.D. et al., 2016. Caractérisation de la variabilité des phénophases de cinq espèces végétales sahéliennes dans la zone nord FERLO, Sénégal. Rev. Ivoirienne Sci. Technol., 27, 117-135.

Diouf M. \& Zaafouri M.S., 2003. Phénologie comparée d'Acacia raddiana au nord et au sud du Sahara. In : Grouzis M. \& Le Floc'h E., éds. Un arbre au désert : Acacia raddiana. Paris : IRD éditions, 103-118.

Fandohan A.B. et al., 2015. Effect of climatic conditions on flowering and fruiting of Tamarindus indica (Fabaceae). J. Hortic. For., 7(8), 186-192, doi.org/10.5897/ jhf2015.0403

Frankie G.W., Baker H.G. \& Opler P.A., 1974. Tropical plant phenology: applications for studies in community ecology. In: Lieth H., ed. Phenology and seasonality modeling. Berlin: Springer-Verlag, 287-296.
Ganglo J.C. \& de Foucault B., 2006. Plant communities, forest site identification and classification in Toffo reserve, South-Benin. Bois For. Trop., 288(2), 25-38.

Grouzis M. \& Sicot M., 1980. Une méthode d'étude phénologique de populations d'espèces ligneuses sahéliennes : influence de quelques facteurs écologiques. In: Le Houérou H.N., éd. Les fourrages ligneux en Afrique : état actuel des connaissances. Addis-Abeba : CIPEA, 231-237.

Harrell F.E. et al., 2018. Hmisc: Harrell Miscellaneous. $R$ package version 4.1-1, https:/CRAN.R-project.org/ package $=$ Hmisc, $(07 / 06 / 2021)$.

Jaouadi W., Hamrouni L. \& Khouja M.L., 2012. Phénologie d'Acacia tortilis subsp. raddiana dans le parc national de Bou Hedma en Tunisie, effet du site sur les phénophases de l'espèce. Bois For. Trop., 312(2), 21-29, doi. org/10.19182/bft2012.312.a20500,

Kouyaté A.M., 2005. Aspects ethnobotaniques et étude de la variabilité morphologique, biochimique et phénologique de Detarium microcarpum Guill. \& Perr. au Mali. Thèse de doctorat : Université de Gand (Belgique).

Lawin I.F. et al., 2019. Connaissances et usages de Cola millenii K. Schum. (Malvaceae) en zones guinéenne et soudano-guinéenne au Bénin. Bois For. Trop., 339, 61-74.

Lawin I.F. et al., 2021. Morphological variability of fruits of Cola millenii K. Schum. from seven phytogeographical districts in Benin: opportunity for domestication. Genet. Resour. Crop Evol., 68, 1225-1242, doi.org/10.1007/ s10722-020-01086-0

Lebourgeois F. et al., 2006. Observations phénologiques des arbres forestiers : concepts, intérêts et problématiques actuelles. RDV Techn., 13, 19-22.

Lobo J.A. et al., 2003. Factors affecting phenological patterns of bombacaceous trees in seasonal forests in Costa Rica and Mexico. Am. J. Bot., 90, 1054-1063, doi. org/10.3732/ajb.90.7.1054

Mahamane A., Saadou M. \& Lejoly J., 2007. Phénologie de quelques espèces ligneuses du Parc national $\mathrm{du} \ll \mathrm{W} » \mathrm{du}$ Niger. Sécheresse, 1E(4), 1-13.

Morellato L.P.C., Camargo M.G.G. \& Gressler E., 2013. A review of plant phenology in South and Central America. In: Schwartz M.D., ed. Phenology: an integrative environmental science. The Netherlands: Springer, 91113.

Natta A.K., 2003. Ecological assessment of riparian forests in Benin: phytodiversity, phytosociology and spatial distribution of tree species. $\mathrm{PhD}$ thesis: Wageningen University and Research (The Netherlands).

Okullo J.B.L., Hall J.B. \& Obua J., 2004 . Leafing, flowering and fruiting of Vitellaria paradoxa subsp. nilotica in savanna parklands in Uganda. Agrofor. Syst, 60, 71-91, doi.org/10.1023/b:agfo.0000009407.63892.99

Oumorou M., 2003. Études écologique, floristique, phytogéographique et phytosociologique des inselbergs du Bénin. Thèse de doctorat: Université libre de Bruxelles (Belgique). 
$\mathrm{R}$ Core Team, 2018. R: a language and environment for statistical computing. Vienna: R Foundation for Statistical Computing, https://www.R-project.org/, (07/06/2021).

Rasamimanana N., Ratsirarson J. \& Richard A.F., 2012. Influence de la variabilité climatique sur la phénologie de la forêt de la Réserve Spéciale de Bezà Mahafaly. Malagasy Nat., 6, 67-82.

Ratovonamana R.Y., Rajeriarison C., $\quad$ Roger E. \& Ganzhorn J.U., 2011. Phenology of different vegetation types in Tsimanampetsotsa National Park, southwestern Madagascar. Malagasy Nat., 5, 14-38.

Rosemartin A.H. et al., 2014. Organizing phenological data resources to inform natural resource conservation. Biol. Conserv., 173, 90-97, doi.org/10.1016/j. biocon.2013.07.003

Seghieri J. \& Simier M., 2002. Variations in phenology of a residual invasive shrub species in Sahelian fallow savannas South-West Niger. J. Trop. Ecol., 18, 897-912, doi.org/10.1017/s0266467402002584

Sinasson Sanni G.K., Shackleton C.M. \& Sinsin B., 2017. Reproductive phenology of two Mimusops species in relation to climate, tree diameter and canopy position in
Benin (West Africa). Afr. J. Ecol., 56(2), 323-333, doi. org/10.1111/aje.12457

Wolkovich E.M., Cook B.I. \& Davies T.J., 2014. Progress towards an interdisciplinary science of plant phenology: building predictions across space, time and species diversity. New Phytol., 201, 1156-1162, doi.org/10.1111/ nph.12599

Yadav R.K. \& Yadav A.S., 2008. Phenology of selected woody species in a tropical dry deciduous forest in Rajasthan, India. Trop. Ecol., 49(1), 25-34.

Yédomonhan H., Hounadagba C.J., Akoègninou A. \& Van der Maesen L.J.G., 2008. Structure et diversité floristique de la végétation des inselbergs du secteur méridional du Centre-Bénin. Syst. Geogr. Plant, 78, 111125.

Zouaoui R. Ksontini M. \& Ferchichi A., 2014. Comparative phenological study of Ziziphus lotus (L.) LAM. in the arid area of Tunisia. Pak. J.Bot., 46(5), 1611-1620.

(40 réf.) 